\title{
Development of articulated robot trajectory planning
}

\section{Zhe Li, Gongfa Li, Ying Sun*, Guozhang Jiang and Jianyi Kong}

College of Machinery and Automation,

Wuhan University of Science and Technology,

Wuhan, 430080, China

Email: 1653485877@qq.com

Email: ligongfa@wust.edu.cn

Email: 493530316@qq.com

Email:whjgz@wust.edu.cn

Email: 15697188659@wo.com.cn

*Corresponding author

\section{Honghai Liu}

State Key Laboratory of Mechanical System and Vibration,

School of Mechanical Engineering,

Shanghai Jiao Tong University,

Shanghai; 200240, China

and

Intelligent Systems and Biomedical Robotics Group,

School of Computing,

University of Portsmouth,

Portsmouth, PO1 3HE, UK

Email: honghai.liu@sjtu.edu.cn

Email: honghai.liu@port.ac.uk

\begin{abstract}
Articulated robot is now widely employed in manufacture, such as, welding, painting, and assembly, with high precision and endurance. It plays an important role in scientific and technological innovation. Trajectory planning of articulated robot is one of the key researches in industrial robot. The commonly used trajectory planning algorithm of articulated robot, such as, polynomial interpolation algorithm in joint space and linear interpolation in Cartesian space are introduced. Researches on articulated robot trajectory planning are surveyed. Meanwhile these articulated robot trajectory planning algorithms are analysed. Some further researches and developing trend of articulated robot trajectory planning are indicated.
\end{abstract}

Keywords: articulated robot; trajectory planning; joint space; Cartesian space.

Reference to this paper should be made as follows: Li, Z., Li, G., Sun, Y., Jiang, G., Kong, J. and Liu, H. (2017) 'Development of articulated robot trajectory planning', Int. J. Computing Science and Mathematics, Vol. 8, No. 1, pp.52-60. 
Biographical notes: Zhe Li received his BS in Mechanical Engineering and Automation from Wuhan University of Technology Huaxia College, Wuhan, China. He is currently occupied in his MS in Mechanical Design and Theory at Wuhan University of Science and Technology. His current research interests include mechanical $\mathrm{CAD} / \mathrm{CAE}$, signal analysis and processing.

Gongfa $\mathrm{Li}$ received his $\mathrm{PhD}$ degree in Wuhan University of Science and Technology, Wuhan, China. He is currently a Professor in Wuhan University of Science and Technology. His major research interests are computer aided engineering, mechanical $\mathrm{CAD} / \mathrm{CAE}$, modelling and optimal control of complex industrial process.

Ying Sun is currently an Associate Professor in Wuhan University of Science and Technology. Her major research focuses on teaching research in mechanical engineering.

Guozhang Jiang received his $\mathrm{PhD}$ degree in Wuhan University of Science and Technology, China. He is currently a Professor in Wuhan University of Science and Technology. His research interests are computer aided engineering, mechanical CAD/CAE and industrial engineering and management system.

Jianyi Kong received his PhD degree in Helmut Schmidt Universitat, Germany. $\mathrm{He}$ is currently a Professor in Wuhan University of Science and Technology. His research interests are intelligent machine and controlled mechanism, mechanical and dynamic design and fault diagnosis of electrical system, mechanical CAD/CAE, intelligent design and control.

Honghai Liu received his $\mathrm{PhD}$ degree in Intelligent Robotics from Kings College, University of London, London, UK. He is currently a Professor of School of Mechanical Engineering in Shanghai Jiao Tong University, Shanghai, China and a Professor of Intelligent Systems in Portsmouth University, Portsmouth, UK. His research interests are approximate computation, pattern recognition, multi-sensor based information fusion and analytics, human machine systems, advanced control, intelligent robotics and their practical applications.

\section{Introduction}

Articulated robot, also known as joint arm robot or joint mechanical arm, is one of the most common form of industrial robot now (Gasparetto and Zanotto, 2010). The main characteristic of articulated robot is to imitate the basic structure of human's arm and waist, so body structure includes frame structure, the waist joints, rotating device, large arm structure, large joints rotating device, forearm rotation device, wrist and forearm joint, wrist joint rotation device, and the end executor (Abdel-Malek et al., 2006). Articulated robot has larger relative space and absolute work space than other robot under the condition of same size (Liu et al., 2013). It has a high degree of freedom, suitable for almost any work with different track or angle. Automatic work can be done by robot free programming. It can improve production efficiency, and can control error rate. It is suitable for mechanical automation operation in many industrial fields, such as, automatic assembling, spray paint, handling, welding. 
The purpose of tractor planning is to calculate the expected trajectory, namely, to describe the robot's task, motion path, and trajectory, and to real-time calculate the displacement, velocity and acceleration of the robot (Gasparetto and Zanotto, 2008). Articulated robot trajectory planning is the key to the development and application of robots is also research hotspot in the field of robot in recent years (Kohrt et al., 2013). Articulated robot trajectory planning plays an important role in the control of the robot, its performance has a decisive significance to the robot's working efficiency, running smoothness and energy consumption (Chettibi et al., 2004). In actual production application robot mechanical system should be stable and no vibration, as far as possible to avoid mutations of displacement, velocity and acceleration in the process of movement. Mutation of motion requires a great deal of power. Motor limited by physical condition, cannot provide so much energy. It will cause the wear of robot's joints and reduce service life of the robot (Yong et al., 2005). To meet these requirements that the robot's each joint can not only make the smooth movement without vibration, and can achieve the goal of high efficiency and energy saving, the appropriate method for robot trajectory planning must be selected. When articulated robot works the shape of related trajectory should be analysed, related information should be acquired and input into control system to control the robot to work (Cesare and Sergio, 2013). Articulated robot trajectory planning as an important module in the control system is significant for motion stability of articulated robot. However, researches on articulated robot trajectory planning are rarely surveyed. Therefore, researches on articulated robot trajectory planning are systematically analysed in this paper. Meanwhile these articulated robot trajectory planning algorithms are compared. Some further researches and developing trend of articulated robot trajectory planning are indicated.

\section{Trajectory planning in joint space}

The joint variables are expressed as a function of time, and its first and second order time derivative are planned when trajectory planning is in joint space (Elnagar and Hussein, 2000). Joint space method does not consider the shape of path between two points, and its calculation is not complex. Because the corresponding relationship between joint space and Cartesian space is not continuous, it would not exist singularity problem (Liu et al., 2010). Commonly used method (polynomial interpolation algorithm) is described below.

In the process of robot movement, if the beginning and end position of end-effector is known, two corresponding position's joint angle can be obtained by inverse kinematics equations (Piazzi and Visioli, 2000). The trajectory of end-effector in joint space can be decrypted by the start and end points of joint angle's smooth trajectory function $\theta(t)$. To achieve the smooth movement of the robot, the trajectory of each joint function $\theta(t)$ need to meet at least four constraint conditions, namely, the two endpoint location and speed of two endpoint constraints. Endpoint location constraint refers to the joint angle of the starting position and end position. The initial value is the initial joint angle $\theta_{0}$, and the terminal value is terminal joint angle $\theta_{f}$, namely

$$
\left.\begin{array}{l}
\theta(0)=\theta_{0} \\
\theta\left(t_{f}\right)=\theta_{f}
\end{array}\right\}
$$


To meet the requirements of continuity of the joint movement speed, start and end points of joint speed can be set to zero, namely,

$$
\left.\begin{array}{l}
\theta(0)=0 \\
\theta\left(t_{f}\right)=0
\end{array}\right\}
$$

Given above four constraints can uniquely identify a cubic polynomial:

$$
\theta(t)=a_{0}+a_{1} t+a_{2} t^{2}+a_{3} t_{3}
$$

In the process of movement joint velocity and acceleration are as follows:

$$
\left.\begin{array}{l}
\dot{\theta}(t)=a_{1}+2 a_{2} t+3 a_{3} t^{2} \\
\ddot{\theta}(t)=2 a_{2}+6 a_{3} t
\end{array}\right\}
$$

The joint movement's initial and terminal speed is zero. And its cubic polynomial interpolation function that meets the requirements of continuous smooth movement:

$$
\theta(t)=\theta_{0}+\frac{3}{t_{f}^{2}}\left(\theta_{f}-\theta_{0}\right) t^{2}-\frac{3}{t_{f}^{3}}\left(\theta_{f}-\theta_{0}\right) t^{3}
$$

The joint angular velocity and angular acceleration expression is:

$$
\begin{aligned}
& \dot{\theta}(t)=\frac{6}{t_{f}^{2}}\left(\theta_{f}-\theta_{0}\right) t-\frac{9}{t_{f}^{3}}\left(\theta_{f}-\theta_{0}\right) t^{2} \\
& \ddot{\theta}(t)=\frac{6}{t_{f}^{2}}\left(\theta_{f}-\theta_{0}\right) t-\frac{18}{t_{f}^{3}}\left(\theta_{f}-\theta_{0}\right) t
\end{aligned}
$$

\section{Trajectory planning in Cartesian space}

In certain cases, the trajectory shape of the end-effector have to meet specific requirements, there have various shapes of trajectory except simple straight trajectory between different points (Plessis and Snyman, 2003). It is needed to plan trajectory for robot in Cartesian space. In fact, all of the methods used in joint space trajectory planning can be used in Cartesian space trajectory planning. The most essential difference is that Cartesian space trajectory planning requires solving inverse kinematics equation to calculate the joint angle repeatedly, by computing the inverse Jacobi matrix to solve the joint angular velocity of the robot. Function obtained in the Cartesian space trajectory planning is posture of the robot end-effector, a lot of inverse solution of kinematics solution is needed so that it can be converted into joint value (Koen et al., 2014). Commonly used method (linear interpolation algorithm) is described below.

The starting point of the straight line is known to find position of the middle point (interpolation point) (Saramago and Ceccareli, 2002). For most of the robot, its position remains the same in the movement along a straight line. Linear interpolation steps are as follows: 
1 The coordinate location of starting point is $p_{0}\left(x_{0}, y_{0}, z_{0}\right), p_{f}\left(x_{f}, y_{f}, z_{f}\right)$, where $p_{0}$ and $p_{f}$ are relative coordinate system. Calculate the length

$$
L=\sqrt{\left(x_{f}-x_{0}\right)^{2}+\left(y_{f}-y_{0}\right)^{2}+\left(z_{f}-z_{0}\right)^{2}} \text {. }
$$

2 The interval trip is divided into several ways, such as, uniform speed, acceleration, deceleration to discuss:

a uniform speed: if the speed is $v$, the trip in interpolation period $T_{s}$ is $d_{1}=v T_{s}$

$\mathrm{b}$ acceleration: if the acceleration is $a$ and starting speed is $v_{0}$, the trip in interpolation period $T_{s}$ is $d_{2}=v_{0} T_{s}+\frac{1}{2} a T_{s}^{2}$, the distance in the entire acceleration is $s=\frac{1}{2 a} v_{0}^{2}$, and the time is $t_{2}=\frac{v_{0}}{a}$

c deceleration: if the acceleration is $a$ and starting speed is $v_{1}$, the trip in interpolation period $T_{s}$ is $d_{2}=v_{1} T_{s}-\frac{1}{2} a T_{s}^{2}$, the distance in the entire acceleration is $s=\frac{1}{2 a} v_{1}^{2}$, and the time is $t_{3}=\frac{v_{1}}{a}$.

3 Calculate total time $t=t_{1}+t_{2}+t_{3}$. calculate interpolation points $N=\frac{t}{T_{S}}$.

4 Judge which period interpolation point in (the accelerating period, uniform section, or deceleration section), determine the increment of each shaft, real-time computing the interpolation point coordinates value.

5 According to the coordinate values, using inverse kinematics to calculate each joint angle.

6 Interpolation calculation of joint angle.

The robot realised a space trajectory (trajectory discrete points). These discrete points are very close to each other and the robot trajectory with sufficient precision to approximate trajectory (Rubio et al., 2012).

In order to ensure the density of discrete points, time interpolation and distance interpolation can use.

\section{Development of trajectory planning}

The point-to-point movement problem of robot optimal trajectory planning is studied by literature (Saramago and Steffen, 2000) under the premise of hypothesis and approximate. The trajectory in joint space is planned by cubic polynomial method in literature (Rubio et al., 2010). There has a diagonal equation to solve. When solving the equation if the intermediate nodes of the trajectory increases will cause the increase of calculation in the process of solving. The trajectory planning based on cubic spline function which optimises the running time of the trajectory has realised online implementation in literature (Saramago and Steffen, 2001). But the acceleration continuity between the path points cannot be guaranteed. The time optimal trajectory 
planning based on cubic spline function is studied in literature (Carlos et al., 2015). The pulse optimal trajectory planning based on cubic spline function is studied in literature (Saravanan et al., 2009). The minimum pulse and pulse continuous trajectory planning method is proposed in literature (Boryga, 2014; Valero et al., 2006). The method can avoid vibration of mechanical arm and improve the trajectory tracking precision. The kinematic method is used for robot time optimal trajectory planning in literature (Mansouri et al., 2008). The time optimal trajectory planning that the limit of maximum joint torque is instead by the limit of joint acceleration is studied in literature (Masehian and Amin-Naseri, 2004). The method can decrease the difficulty of problem, but it still exist error. Because the hyperbolic function method is simple, the robot trajectory in joint space is planned by the method in literature (Marek et al., 2015). But hyperbolic function also has its disadvantages, namely, when determining the parameters it needs a lot of experience. It puts forward a new time optical method in literature (Pires et al., 2009). The constraint of end-effector's path, joint speed, and drive torque is considered in the method. These constraints are transferred to the path parameter space by the method that parameterised system is dynamic close to a solution space. And then the model of DAE is established for the trajectory planning. Dynamic programming algorithms are introduced in literature (Peng-Cheng et al., 2014; Brogardh, 2007). A kind of non-strict time optimal trajectory planning method is put forward in literature (Piltan et al., 2011; Chong et al., 2009). The driver's acceleration is limited in this method. The trajectory obtained by the method can be implemented effectively. For example, spline function (such smooth trajectory) is widely used in kinematic and dynamic trajectory planning in the literature to get the trajectory with its acceleration continuous. A time optimal online trajectory planning method is proposed in literature (Liu et al., 2013). The dynamics constraints of mechanical arm are considered. Given path points are made dynamics calculation, which can determine the kinematics constraints of mechanical arm. This method improves the traditional industrial robot trajectory planning method. Interpolation method based on B-spline curve for trajectory planning is proposed in literature (Lieberknecht et al., 2009). The algorithms based on curve optimum above are local optimum. The algorithm proposed in literature (Ghasemi et al., 2012) makes up for the shortcoming of local optimum algorithm based on curve, the global optimal results are obtained. A novel robot trajectory planning method (hybrid cubic B-spline curve convex optimisation method) is proposed in literature (Verscheure et al., 2009), this method can get smooth joint trajectory. The time optimal method based on the simple iterative of drive torque and transmission limit is proposed in literature (Ahn et al., 2012). An indirect control strategy of open-loop optimal is used in this method, and the optimal control problem is transformed into a nonlinear two-point boundary value problem.

\section{Conclusions and prospects}

The articulated robot trajectory planning can either be in joint space or in Cartesian space, but the trajectory planning function must be continuous and smooth and make movement of robot stable and no-quiver. B-spline curve gradually has been widely used because of the advantages of derivative continuity, piecewise, small joint displacement rate, and local support. On the basis of this, different optimisation algorithms are used in related kinematic and dynamic constraints to optimise the robot's movement trajectory. In recent years, intelligent algorithms are used to solve the problem of robot trajectory 
planning; it becomes the research focus in this field. Such as, simulated annealing, genetic algorithm, neural network, ant colony algorithm, particle swarm optimisation (PSO) algorithm, and other intelligent algorithms have been successively applied in robot trajectory planning problem.

\section{Acknowledgements}

This work was supported by Grants of National Natural Science Foundation of China (Grant Nos. 51575407, 51575338, 1575412, 61273106).

\section{References}

Abdel-Malek, K., Mi, Z., Yang, J. and Nebel, K. (2006) 'Optimization-based trajectory planning of the human upper body', Journal of Parallel and Distributed Computing, Vol. 24, No. 6, pp.683-696.

Ahn, J.S., Chung, W.J. and Jung, C.D. (2012) 'Realization of orientation interpolation of 6-axis articulated robot using quaternion', Journal of Central South University, Vol. 19, No. 12, pp.3407-3421.

Boryga, M (2014) 'Trajectory planning of an end-effector for path with loop', Strojniški vestnik - Journal of Mechanical Engineering, Vol. 60, No. 12, pp.804-814.

Brogardh, T. (2007) 'Present and future robot control development-an industrial perspective', Annual Reviews in Control, Vol. 31, No. 1, pp.69-79.

Carlos, L.A., Francisco. R. and Francisco, V. (2015) 'Improving productivity using a multi-objective optimization of robotic trajectory planning', Journal of Business Research, Vol. 68, No. 7, pp.1429-1431.

Cesare, R. and Sergio, S. (2013) 'Robot trajectory planning by assigning positions and tangential velocities', Robotics and Computer-Integrated Manufacturing, Vol. 29, No. 1, pp.139-156.

Chettibi, T., Lehtihet, H., Haddad, M. and Hanchi, S. (2004) 'Minimum cost trajectory planning for industrial robots', European Journal of Mechanics e A/Solids, Vol. 23, No. 4, pp.703-715.

Chong, J.W.S., Ong, S.K., Nee, A.Y.C. and Youcef-Youmi, K. (2009) 'Robot programming using augmented reality: an interactive method for planning collision-free paths', Robotics and Computer-Integrated Manufacturing, Vol. 25, No. 3, pp.689-701.

Elnagar, A. and Hussein, A. (2000) 'On optimal constrained trajectory planning in 3D environments', Robotics and Autonomous Systems, Vol. 33, No. 4, pp.195-206.

Gasparetto, A. and Zanotto, V. (2008) 'A technique for time-jerk optimal planning of robot trajectories', Robotics and Computer-Integrated Manufacturing, Vol. 24, No. 3, pp.415-426.

Gasparetto, A. and Zanotto, V. (2010) 'Optimal trajectory planning for industrial robots', Adv. Eng. Softw., Vol. 41, No. 4, pp.548-556.

Ghasemi, M.H., Kashiri, N. and Dardel, M. (2012) 'Time-optimal trajectory planning of robot manipulators in point-to-point motion using an indirect method', Proceedings of the Institution of Mechanical Engineers, Part C: Journal of Mechanical Engineering Science, Vol. 22, pp.473-484.

Koen, P., Wim, D., Karel, V.E., Karel, K. and Peter, S. (2014) 'Energy efficient trajectories for an industrial ABB robot', Procedia CIRP, No. 15, pp.105-110.

Kohrt, C., Kohrt, V., Zanotto, A.G. Pipe, Kiely, J. and Schiedermeier, G. (2013) 'An online robot trajectory planning and programming support system for industrial use', Robotics and Computer-Integrated Manufacturing, Vol. 29, No. 1, pp.71-79. 
Lieberknecht, S., Benhimane, S., Meier, P. and Navab, N. (2009) 'A dataset and evaluation methodology for template-based tracking algorithms', Proceedings of the International Symposium on Mixed and Augmented Reality, Orlando, FL, pp.145-51.

Liu, H., Lai, X. and Wu, W. (2013) 'Time-optimal and jerk continuous trajectory planning for robot manipulators with kinematic constraints', Rob. Comput. Integr. Manuf., Vol. 29, No. 2, pp.309-317.

Liu, Z., Bu, W. and Tan, J. (2010) 'Motion navigation for arc welding robots based on feature mapping in a simulation environment', Robotics and Computer-Integrated Manufacturing, Vol. 26, No. 2, pp.137-44.

Mansouri, M., Aliyari Shoorehdeli, M. and Teshnehlab, M. (2008) 'Integer GA for mobile robot path planning with using another GA as repairing function', 2008 IEEE International Conference on Automation and Logistics, pp.135-140.

Marek, B., Andrzej, G., Paweł, K., Krzysztof, G. and Zbigniew, S. (2015) 'Trajectory planning with obstacles on the example of tomato harvest', Agriculture and Agricultural Science Procedia, Vol. 7, pp.27-34.

Masehian, E. and Amin-Naseri, M.R. (2004) 'A voronoi diagram-visibility graph-potential field compound algorithm for robot path planning', In: Journal of Robotic Systems, Vol. 21, No. 6, pp.275-300.

Peng-Cheng, W., Yong-Chun, F. and Yong-Chun, J. (2014) 'A direct swing constraint-based trajectory planning method for underactuated overhead cranes', Acta Automatica Sinica, Vol. 40, No. 11, pp.2414-2419.

Piazzi, A. and Visioli, A. (2000) 'Global minimum-jerk trajectory planning of robot manipulators', IEEE Transactions on Industrial Electronics, Vol. 47, No. 1, pp.140-149.

Piltan, F., Haghighi, S.H.T. and Sulaiman, N. (2011) 'Artificial control of PUMA robot manipulator:a-review of fuzzy inference engine and application to classical controller', International Journal of Robotics and Automation, Vol. 2, No. 5, pp.401-425.

Pires, J.N., Veiga, G. and Araujo, R. (2009) 'Optimal trajectory planning for industrial robots', $A d v$. Eng. Softw., Vol. 36, No. 1, pp.73-83.

Plessis, L.D. and Snyman, J. (2003) 'Trajectory-planning through interpolation by overlapping cubic arcs and cubic splines', International Journal for Numerical Methods in Engineering, Vol.57, No. 11, pp.1615-1641.

Rubio, F.J., Valero, F.J., Suñer, J.L. and Cuadrado, J. (2012) 'Optimal time trajectories for industrial robots with torque, power, jerk and energy consumed constraints', Industrial Robot: An International Journal, Vol. 39, No. 1, pp.92-100.

Rubio, F.J., Valero, F.J., Suñer, J.L. and Garrido, A. (2010) 'The simultaneous algorithm and the best interpolation function for trajectory planning', Industrial Robot: An International Journal, Vol. 37, No. 5, pp.441-451.

Saramago, S. and Ceccareli, M. (2002) 'Trajectory modelling of robot manipulators in the presence of obstacles', Robotica, Vol. 20, No. 4, pp.395-404.

Saramago, S. and Steffen, V. (2000) 'Optimal trajectory planning of robot manipulators in the presence of moving obstacles', Mechanism and Machine Theory., Vol. 35, No. 8, pp.1079-1094.

Saramago, S. and Steffen, V. (2001) 'Trajectory modelling of robot manipulators in the presence of obstacles', Journal of Optimization Theory and Applications, Vol. 110, No. 1, pp.17-34.

Saravanan, R., Ramabalan, S. and Balamurugan, C. (2009) 'Evolutionary multi-criteria trajectory modeling of industrial robots in the presence of obstacles', Engineering Applications of Artificial Intelligence, Vol. 22, No. 2, pp.329-342.

Valero, F., Mata, V. and Besa, A. (2006) 'Trajectory planning in workspaces with obstacles taking into account the dynamic robot behaviour', Mechanism and Machine Theory, Vol. 41, No. 5, pp.525-536. 
Verscheure, D., Demeulenaere, B., Swevers, J., Schutter, J.D. and Diehl, M. (2009) 'Time-optimal path tracking for robots: a convex optimization approach', IEEE Trans. Automat. Contr., Vol. 54, No. 10, pp.2318-2327.

Yong, J., MingYang, Z., HongGuang, W. and LiJing, F. (2005) 'Hybrid navigation for a climbing robot by fuzzy neural network and trajectory planning', 2005 International Conference on Machine Learning and Cybernetics, Vol. 2, pp.1069-1075. 